\title{
Solution of MHD Flow past a Vertical Porous Plate through a Porous Medium under Oscillatory Suction
}

\author{
Ashraf A. Moniem ${ }^{1}$, W. S. Hassanin ${ }^{2}$ \\ ${ }^{1}$ University College in Al-Jamoum, Umm Al-Qura University, Mecca, KSA \\ ${ }^{2}$ Akhbar El-Yom Academy, Giza, Egypt \\ Email: a_saltah@hotmail.com, ha_ssanin@yahoo.co \\ Received September 22, 2012; revised February 21, 2013; accepted February 28, 2013
}

Copyright (C) 2013 Ashraf A. Moniem, W. S. Hassanin. This is an open access article distributed under the Creative Commons Attribution License, which permits unrestricted use, distribution, and reproduction in any medium, provided the original work is properly cited.

\begin{abstract}
The model of mass transfer on free convective flow of a viscous incompressible electrically conducting fluid past vertically porous plate through a porous medium with time dependant permeability and oscillatory suction in presence of a transverse magnetic field is considered. Perturbation technique is obtained the solution for velocity field and concentration distribution analytically. The effects of the flow parameters on the velocity field and concentration distribution are presented with the aid of figures. Also, the skin friction and the rate of mass transfer are calculated with the aid of tables.
\end{abstract}

Keywords: MHD Flow; Mass Transfer; Porosity; Oscillation Suction

\section{Introduction}

The phenomena of MHD flow with unsteady oscillatory free convective flows play an important role in aerospace technology and in chemical engineering turbo-machinery. Such flow arise either due to unsteady motion of boundary or due to oscillatory free system velocity. Several authors have studied the free convection and mass transfer flow of a viscous fluid through porous medium. They considered that, the permeability of the porous medium is assumed to be constant, while the porosity of the medium may be not necessary constant because the porous material containing the fluid is non-homogenous medium. Then, the permeability of the porous medium may be not necessary constant. The effect of mass transfer on free convective hydromagnetic oscillatory flow past an infinite vertical unsteady flow of non-Newtonian fluid has been estimated by Koullias et al. [1]. Ashgar et al. [2] have reported the periodic unsteady flows of non-Newtonian fluid. The flow of non-Newtonian fluid induced due to the oscillations of a porous plate has studied by Hayat et al. [3]. Singh and Gupta [4] have investigated the MHD free convective flow of a viscous fluid through a porous medium bounded by an oscillating porous plate in the slip flow regime with mass transfer. Das et al. [5] analyzed the mass transfer effects on unsteady flow past an accelerated vertical porous plate with suction employing numerical methods. Raptis [6] analyzed the unsteady free convection flow through a porous medium. Hossain and Begum [7] discussed the effect of mass transfer and free convection on the flow past a vertical plate. Unsteady hydromagnetic convective flow past an infinite vertical porous flat plate in a porous medium has been discussed by Das and his coworkers [8]. The objective of the present study is to analyzed the effects of permeability variation and oscillatory suction velocity in free convective and mass transfer flow of a viscous incompressible fluid past an infinite vertical porous plate through a porous medium when the plate is subjected to a time dependent suction velocity normal to the plate in the presence of a uniform transverse magnetic field. The solutions for velocity field and concentration distribution are obtained by perturbation technique analytically. The effects of the flow parameters on the velocity field and concentration distribution are presented with the aid of figures. Also, the skin friction and the rate of mass transfer are calculated with the aid of tables.

\section{Mathematical Analysis}

The unsteady free convective flow of a viscous incompressible electrically conducting fluid past an infinite 
vertical porous plate through a porous medium with time dependant permeability and oscillatory suction in presence of a transverse magnetic field is considered. The $y$-axis is taken along the infinite vertical porous plate and $x$-axis is perpendicular to it and the velocity in this direction is $u$. In view of these, the analysis of the problem is based on the following assumptions:

- All the fluid properties are constant;

- The plate as well as the fluid is assumed to be at the same temperature and the concentration of the species is raised or lowered;

- The magnetic Reynolds number is small so that the induced magnetic field can be neglected in comparison to the applied magnetic field;

- The permeability of the porous medium is assumed to be $K^{\prime}\left(t^{\prime}\right)=K_{p}\left(1+\varepsilon \mathrm{e}^{\mathrm{i} \omega^{\prime} t^{\prime}}\right)$;

- The suction velocity is assumed to be $v\left(t^{\prime}\right)=-v_{\circ}\left(1+\varepsilon \mathrm{e}^{\mathrm{i} \omega^{\prime} t^{\prime}}\right)$.

With foregoing assumptions and taking the usual Boussinesq's approximation into account as well as following non-dimensional quantities:

$$
\begin{aligned}
& u=\frac{u}{v_{\circ}}, y=\frac{v_{\circ} y^{\prime}}{v}, t=\frac{v_{\circ}^{2} t^{\prime}}{4 v}, S_{c}=\frac{v}{D}, \omega=\frac{4 v \omega^{\prime}}{v_{\circ}^{2}}, \\
& K_{p}=\frac{v^{2} K^{\prime}}{v_{\circ}^{2}}, M^{2}=\frac{\sigma v B_{\circ}^{2}}{\rho v_{\circ}^{2}}, C=\frac{C^{\prime}-C_{\infty}}{C_{w}-C_{\infty}}, \\
& G_{c}=\frac{\left(C_{w}-C_{\infty}\right) v g \beta}{v_{\circ}^{3}} .
\end{aligned}
$$

Therefore, the non-dimensional form of the governing equations for momentum and concentration are:

$$
\begin{gathered}
u_{y y}+f(t) u_{y}+C G_{c}=\frac{u_{t}}{4}+\left(M^{2}+\frac{1}{f(t)}\right) u, \\
C_{y y}+f(t) S_{c} C_{y}=\frac{S_{c} C_{t}}{4},
\end{gathered}
$$

where $f(t)=\left(1+\varepsilon \mathrm{e}^{\mathrm{i} \omega t}\right) \quad$ Subjected to the following boundary conditions:

$$
\begin{aligned}
& u(0, t)=C(0, t)=f(t), \text { and } \\
& \lim _{y \rightarrow \infty} u(y, t)=\lim _{y \rightarrow \infty} C(y, t)=0 .
\end{aligned}
$$

\section{Solutions of the Problem}

In order to solve the problem, we assume the solutions of the following form because the amplitude $\varepsilon(\ll 1)$ of the permeability variation is very small:

$$
\begin{gathered}
u(y, t)=u_{\circ}(y)+\varepsilon u_{1}(y) \mathrm{e}^{\mathrm{i} \omega t}, \\
C(y, t)=C_{\circ}(y)+\varepsilon C_{1}(y) \mathrm{e}^{\mathrm{i} \omega t} .
\end{gathered}
$$

Substitute Equations (5) and (6) into Equations (2) and
(3) and equate the harmonic and non-harmonic terms to obtain the following non-dimensional ordinary differential equations:

$$
\begin{gathered}
u_{\circ}^{\prime \prime}+u_{\circ}^{\prime}+G_{c} C_{\circ}=a_{1} u_{\circ}, \\
u_{1}^{\prime \prime}+u_{1}^{\prime}+u_{\circ}^{\prime}+\frac{u_{\circ}}{K_{p}}+G_{c} C_{1}=a_{2} u_{1}, \\
C_{\circ}^{\prime \prime}+S_{c} C_{\circ}^{\prime}=0, \\
C_{1}^{\prime \prime}+S_{c} C_{1}^{\prime}+S_{c} C_{\circ}^{\prime}=\frac{\mathrm{i} \omega}{4} S_{c} C_{1} .
\end{gathered}
$$

where $a_{1}=M^{2}+\frac{1}{k_{p}}$ and $a_{2}=a_{1}+\frac{i \omega}{4}$.

Furthermore, the boundary conditions are reduced to

$$
\begin{aligned}
& u_{\circ}(0)=u_{1}(0)=C_{\circ}(0)=C_{1}(0)=1 \text { and } \\
& u_{\circ}=u_{1}=C_{\circ}=C_{1} \rightarrow 0 \text { as } y \rightarrow \infty
\end{aligned}
$$

By solving Equations (7)-(10) under boundary conditions (11), we obtain

$$
\left.\begin{array}{l}
u(y, t) \\
=\mathrm{e}^{-m_{2} y}+\frac{G_{c}\left(\mathrm{e}^{-S_{c} y}-\mathrm{e}^{-m_{2} y}\right)}{\left(m_{2}-S_{c}\right)\left(m_{3}+S_{c}\right)} \\
+\varepsilon \mathrm{e}^{\mathrm{i} \omega t}\left\{\mathrm{e}^{-m_{4} y}+\frac{G_{c}\left(1-\frac{4 \mathrm{i}}{\omega} S_{c}\right)\left(\mathrm{e}^{-m_{1} y}-\mathrm{e}^{-m_{4} y}\right)}{\left(m_{4}-m_{1}\right)\left(m_{5}+m_{1}\right)}\right. \\
+\frac{G_{c}\left(\mathrm{e}^{-S_{c} y}-\mathrm{e}^{-m_{4} y}\right)}{\left(m_{4}-S_{c}\right)\left(m_{5}+S_{c}\right)}\left(\frac{4 \mathrm{i}}{\omega} S_{c}+\frac{\left(\frac{1}{K_{p}}-S_{c}\right)}{\left(m_{2}-S_{c}\right)\left(m_{3}+S_{c}\right)}\right) \\
+\frac{\left(\frac{1}{K_{p}}-m_{2}\right)\left(\mathrm{e}^{-m_{2} y}-\mathrm{e}^{-m_{4} y}\right)(12)}{\left(m_{4}-m_{2}\right)\left(m_{5}+m_{2}\right)}\left(1-\frac{G_{c}}{\left(m_{2}-S_{c}\right)\left(m_{3}+S_{c}\right)}\right)
\end{array}\right\}
$$

The real and imaginary parts are separated from Equations (12) and (13). The physical significance is the real parts which are given as follow:

$$
\begin{aligned}
& u(y, t)=u_{\circ}(y)+\varepsilon\left(M_{r} \cos (\omega t)-M_{i} \sin (\omega t)\right), \\
& C(y, t)=C_{\circ}(y)+\varepsilon\left(L_{r} \cos (\omega t)-L_{i} \sin (\omega t)\right) .
\end{aligned}
$$


For $\omega t=\frac{\pi}{2}$, the transient velocity and concentration distribution are given by

$$
\begin{aligned}
& u\left(y, \frac{\pi}{2 \omega}\right)=u_{\circ}(y)-\varepsilon M_{i}, \\
& C\left(y, \frac{\pi}{2 \omega}\right)=C_{\circ}(y)-\varepsilon L_{i} .
\end{aligned}
$$

The skin friction at the plate in terms of amplitude and phase is given by

$$
\left(\frac{\partial u}{\partial y}\right)_{y=0}=\tau=\tau_{\circ}+\varepsilon|N| \cos (\omega t+\alpha),
$$

where

$$
\begin{aligned}
& \tau_{\circ}=-m_{2}+\frac{G_{c}}{m_{3}+S_{c}},\left(\frac{\mathrm{d} u_{1}}{\mathrm{~d} y}\right)_{y=0}=N=N_{r}+\mathrm{i} N_{i}, \\
& \tan (\alpha)=\frac{N_{i}}{N_{r}} .
\end{aligned}
$$

The Sherwood number (the mass transfer coefficient) at the plate terms of amplitude and phase is given by

$$
-\left(\frac{\partial C}{\partial y}\right)_{y=0}=S_{h}=S_{c}+\varepsilon|Q| \cos (\omega t+\gamma),
$$

where

$$
-\left(\frac{\mathrm{d} C_{1}}{\mathrm{~d} y}\right)_{y=0}=Q=Q_{r}+\mathrm{i} Q_{i}, \tan (\gamma)=\frac{Q_{i}}{Q_{r}} .
$$

The constants $A_{1}-A_{6}, a_{3}, B_{1}-B_{6}, b_{1}-b_{5}, L_{r}$, $L_{i}, M_{r}, M_{i}, m_{1}-m_{5}, N_{1}-N_{12}, N_{r}, N_{i}, Q_{r}, Q_{i}$, $R_{r}$ and $R_{i}$ are defined in appendix section.

\section{Numerical Simulation and Discussion}

The problem of free convective and mass transfer flow of a viscous incompressible electrically conducting fluid past an infinite vertical porous plate through a porous medium when with time dependent permeability and oscillatory suction in presence of a uniform transverse magnetic field has been considered. Perturbation technique is obtained the solution for velocity field and concentration distribution analytically. The effects of the flow parameters such as magnetic parameter $M$, Grashof number for mass transfer $G_{c}$ porosity parameter $K_{p}$ and Schmidt number $S_{c}$ have been studied analytically and presented with the help of Figures 1-4, respectively. The effects of Schmidt number $S_{c}$ on the concentration distribution have been showed in Figure 5. Furthermore, the effects of the flow parameters on skin friction and the rate of mass transfer have been reported with the help of Tables 1-7.

The effect of magnetic field parameter $M$ on the velocity profiles of the flow field keeping other parameters of the flow field constant is showed in Figure 1. The magnetic field parameter is taken in increasing order and it is found to slow down the velocity of the flow field to a considerable amount due to the magnetic pull of the Lorentz force acting on the flow field. This parameter shows overturn effect.

The effect of Schmidt number $S_{c}$ on the velocity profiles of the flow field keeping other parameters of the flow field constant is showed in Figure 2. The Schmidt number is taken in increasing order and it is reveal that the presence of heavier diffusion species has a delaying effect on velocity of the flow field. This parameter shows reverse effect.

The effect of Grashof number for mass transfer $G_{c}$ on the velocity profiles of the flow field keeping other parameters of the flow field constant is showed in Figure 3. It is noted that the Grashof number for mass transfer is accelerated the velocity of the flow field at all points.

The effect of porosity parameter $K_{p}$ on the velocity

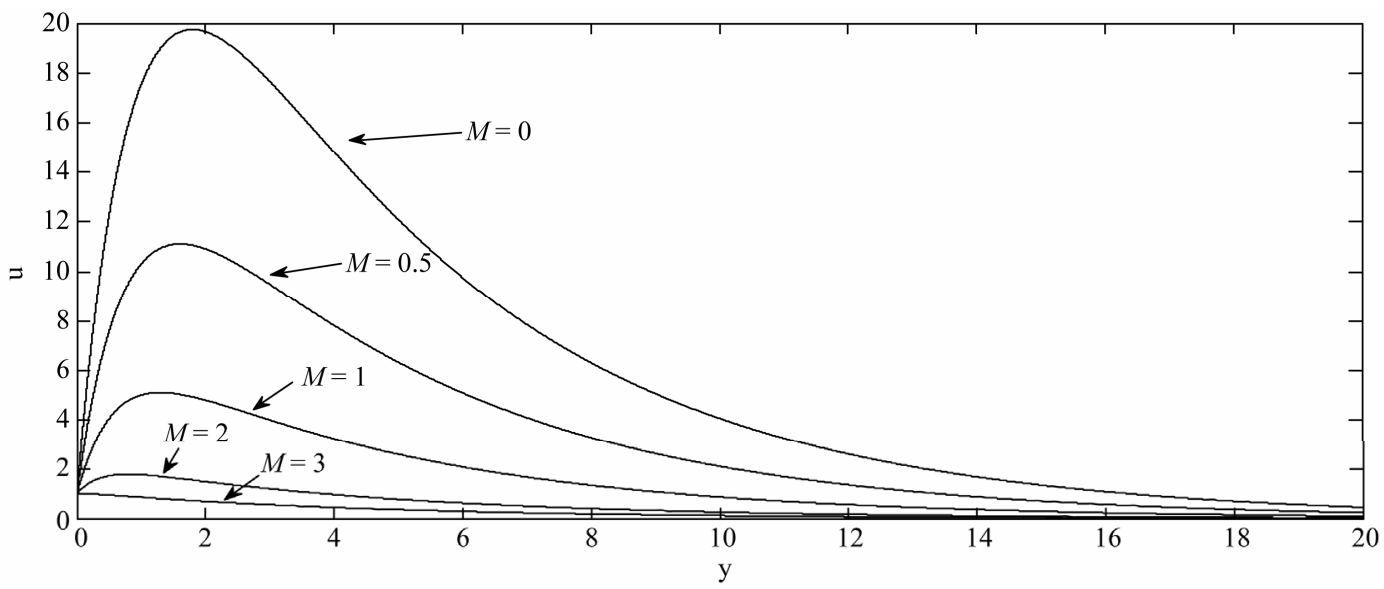

Figure 1. Effect of $M$ on velocity profiles with $G_{c}=10, K_{p}=10, S_{c}=0.22, \varepsilon=0.002, \omega=5$ and $\omega t=\frac{\pi}{2}$. 


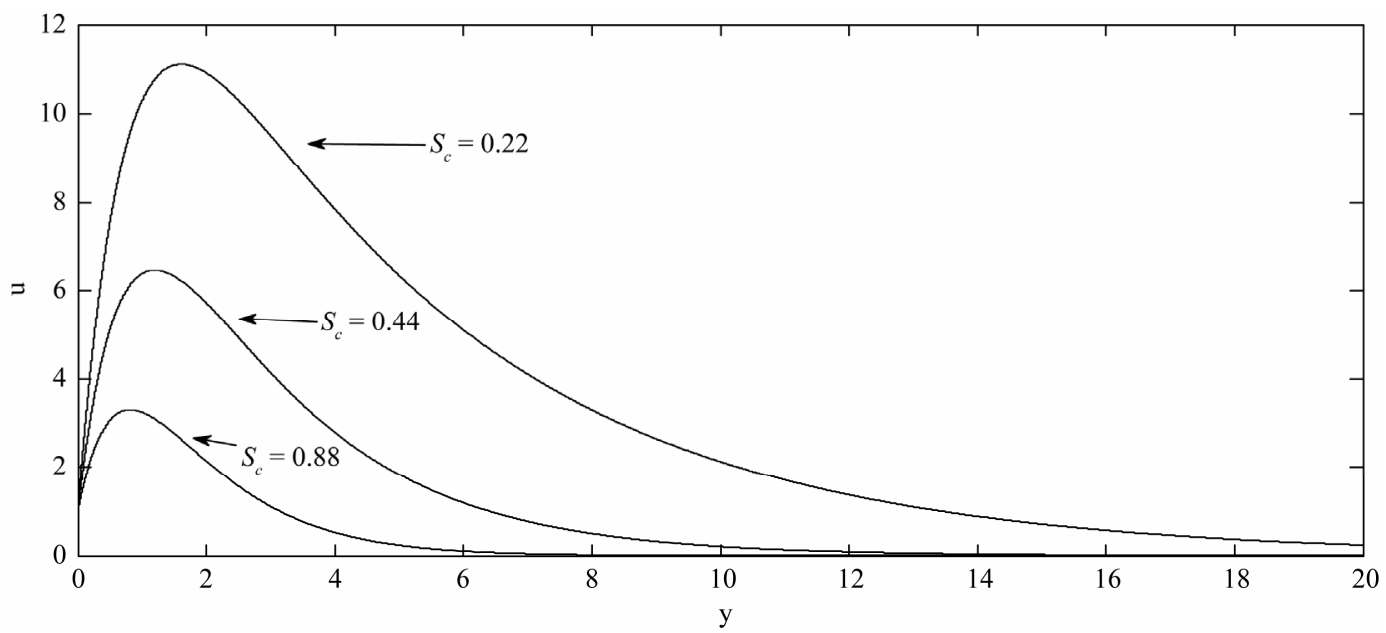

Figure 2. Effect of $S_{c}$ on velocity profiles with $M=0.5, G_{c}=10, K_{p}=10, \varepsilon=0.002, \omega=5$ and $\omega t=\frac{\pi}{2}$.



Figure 3. Effect of $G_{c}$ on velocity profiles with $M=0.5, K_{p}=10, S_{c}=0.22, \varepsilon=0.002, \omega=5$ and $\omega t=\frac{\pi}{2}$.

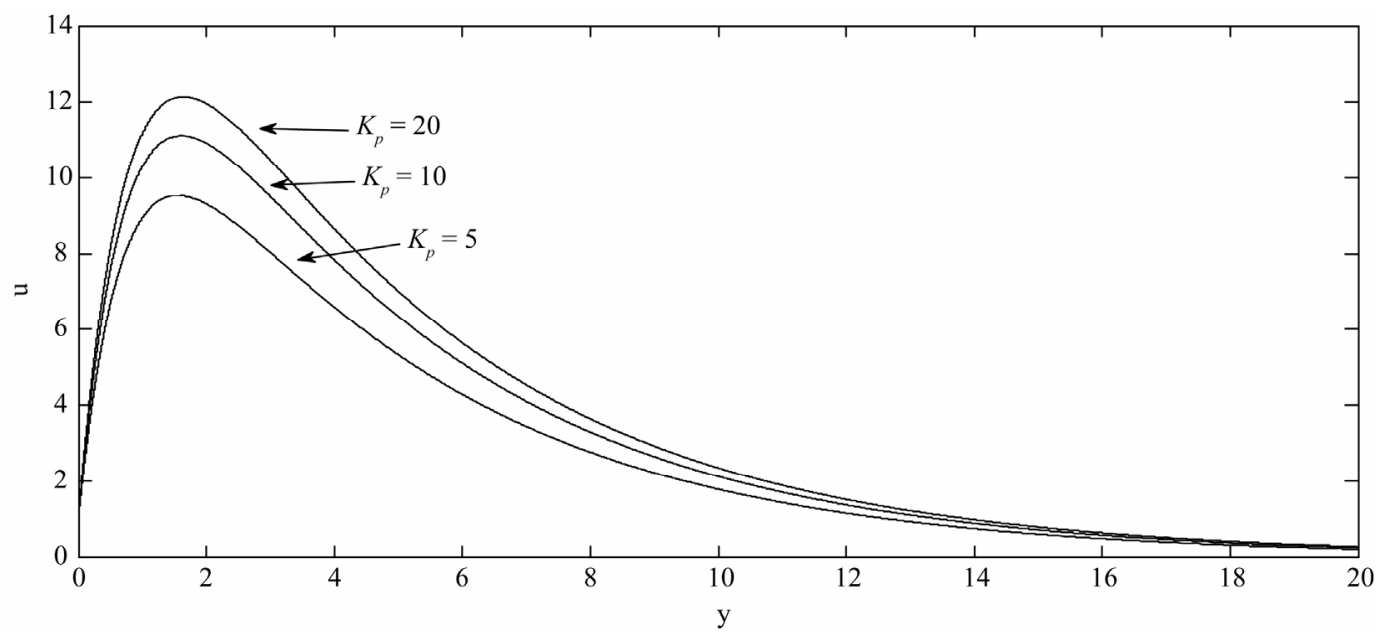

Figure 4. Effect of $K_{p}$ on velocity profiles with $M=0.5, G_{c}=10, S_{c}=0.22, \varepsilon=0.002, \omega=5$ and $\omega t=\frac{\pi}{2}$. 


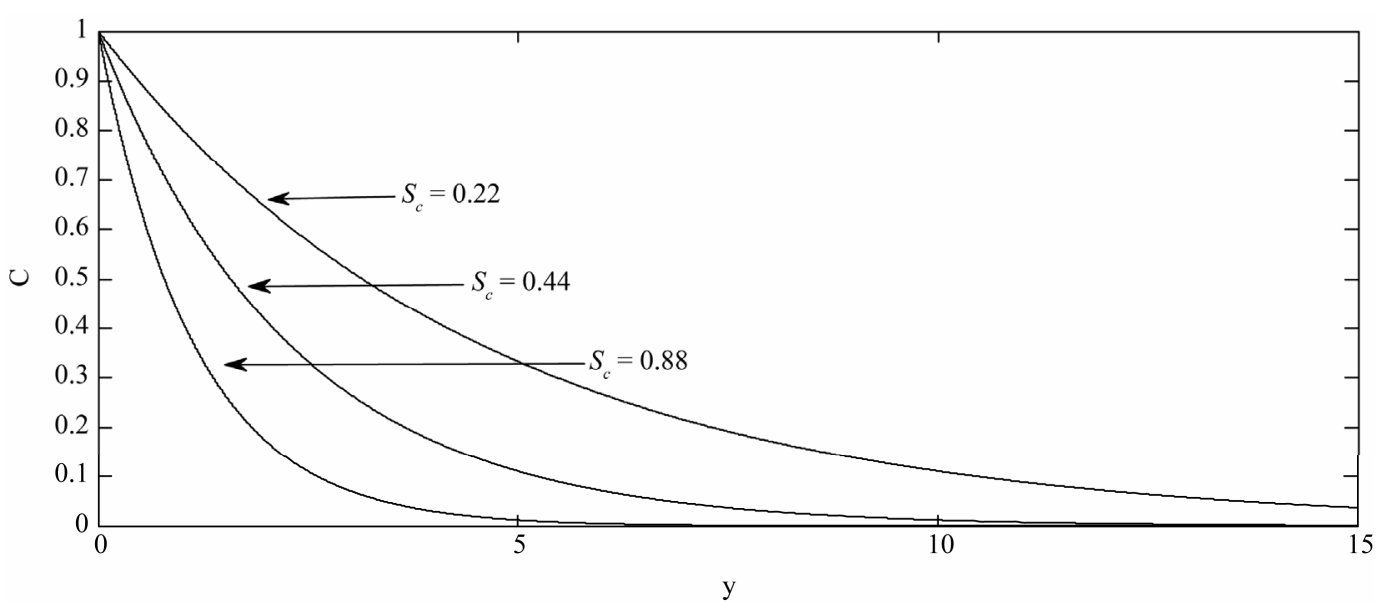

Figure 5. Effect of $S_{c}$ on concentration distribution with $\varepsilon=0.002, \omega=5$ and $\omega t=\frac{\pi}{2}$.

Table 1. Values of amplitude, phase and the skin friction coefficients for different values of $M$ with $G_{c}=10, K_{p}=10$,

\begin{tabular}{ccccc}
$\boldsymbol{S}_{\boldsymbol{c}}=\mathbf{0 . 2 2}, \boldsymbol{\varepsilon}=\mathbf{0 . 0 0 2}, \boldsymbol{\omega}=\mathbf{5}$ and $\boldsymbol{\omega} \boldsymbol{t}=\frac{\boldsymbol{\pi}}{\mathbf{2}}$ & \\
\hline$M$ & $\begin{array}{c}\text { Amplitude } \\
(|N|)\end{array}$ & $\begin{array}{c}\text { Phase } \\
(\tan (\alpha))\end{array}$ & $\tau_{\circ}$ & $\tau$ \\
\hline 0.5 & 15.6917 & -0.5289 & 18.9438 & 18.9586 \\
1 & 9.0363 & -0.5000 & 9.6773 & 9.6854 \\
2 & 2.8416 & -0.4867 & 2.9524 & 2.9549 \\
3 & 0.6636 & 1.5269 & 0.0422 & 0.0433 \\
\hline
\end{tabular}

Table 2. Values of amplitude, phase and the skin friction coefficients for different values of $S_{c}$ with $M=0.5, G_{c}=10$,

\begin{tabular}{|c|c|c|c|c|}
\hline$S_{c}$ & $\begin{array}{c}\text { Amplitude } \\
(|N|)\end{array}$ & $\begin{array}{c}\text { Phase } \\
(\tan (\alpha))\end{array}$ & $\tau_{\text {。 }}$ & $\tau$ \\
\hline 0.22 & 15.6917 & -0.5289 & 18.9438 & 18.9586 \\
\hline 0.44 & 9.6496 & -0.3848 & 12.7193 & 12.7262 \\
\hline 0.66 & 6.2993 & -0.1921 & 9.4252 & 9.4275 \\
\hline 0.88 & 4.4432 & 0.2405 & 7.3864 & 7.3843 \\
\hline
\end{tabular}

profiles of the flow field keeping other parameters of the flow field constant is showed in Figure 4. It is observed that the porosity parameter is enhanced the velocity of the flow field at all points.

The concentration distribution decreases at all points of the flow field with the increase of the Schmidt number $S_{c}$. Singh et al. [9] have applied this result into some foreign mass such as hydrogen $\left(S_{c}=0.22\right)$ helium $\left(S_{c}=0.30\right)$ and ammonia $\left(S_{c}=0.78\right)$.
Table 3. Values of amplitude, phase and the skin friction coefficients for different values of $G_{c}$ with $M=0.5, K_{p}=10$,

\begin{tabular}{ccccc}
$\boldsymbol{S}_{\boldsymbol{c}}=\mathbf{0 . 2 2}, \boldsymbol{\varepsilon}=\mathbf{0 . 0 0 2}, \boldsymbol{\omega}=\mathbf{5}$ and $\boldsymbol{\omega} \boldsymbol{t}=\frac{\boldsymbol{\pi}}{\mathbf{2}}$ & \\
\hline$G_{c}$ & $\begin{array}{c}\text { Amplitude } \\
(|N|)\end{array}$ & $\begin{array}{c}\text { Phase } \\
(\tan (\alpha))\end{array}$ & $\tau_{\circ}$ & $\tau$ \\
\hline 5 & 7.1117 & -0.6758 & 8.8346 & 8.8426 \\
10 & 15.6917 & -0.5289 & 18.9438 & 18.9585 \\
15 & 24.3248 & -0.4893 & 29.0531 & 29.0745 \\
20 & 32.9695 & -0.4709 & 39.1623 & 39.1904 \\
\hline
\end{tabular}

Table 4. Values of amplitude, phase and the skin friction coefficients for different values of $K_{p}$ with $M=0.5, G_{c}=10$, $S_{c}=0.22, \varepsilon=0.002, \omega=5$ and $\omega t=\frac{\pi}{2}$.

\begin{tabular}{ccccc}
\hline$K_{p}$ & $\begin{array}{c}\text { Amplitude } \\
(|N|)\end{array}$ & $\begin{array}{c}\text { Phase } \\
(\tan (\alpha))\end{array}$ & $\tau_{\circ}$ & $\tau$ \\
\hline 5 & 15.0930 & -0.5837 & 16.6276 & 16.6428 \\
10 & 15.6917 & -0.5289 & 18.9438 & 18.9585 \\
15 & 15.9409 & -0.5025 & 19.8990 & 19.9133 \\
20 & 16.0800 & -0.4874 & 20.4212 & 20.4353 \\
\hline
\end{tabular}

In Tables 1-5, the numerical values of skin friction coefficients $\tau_{\circ}, \tau$ in terms of amplitude $(|N|)$ and phase $(\tan (\alpha))$ for different values of $M, S_{c}, G_{c}, K_{p}$ and $\omega$ are entered, respectively. It is noted that the value of the phase $(\tan (\alpha))$ increases due to decrease in $\omega$ while increases due to increase in $M, S_{c}, G_{c}$ and $K_{p}$. Also, It is observed that an increasing in $G_{c}$ and $K_{p}$ leads to an increasing in the value of amplitude $(|N|)$ while an increase of $M, S_{c}$ and $\omega$ leads to a decrease in the value 
of $|N|$. Further, it is showed that the skin friction coefficients $\tau_{\circ}$ and $\tau$ increase due to increase in $G_{c}, K_{p}$ while decrease due to increase in $M, S_{c}$ and $\omega$ with note that $\tau_{\circ}$ is not function on $\omega$.

In Tables 6 and 7, present the numerical values of amplitude $(|Q|)$, phase $(\tan (\gamma))$ and the rate of mass transfer in term of Sherwood number $S_{h}$ due to the variations in the values of $S_{c}$ and $\omega$, respectively. It is watched that an increase in $S_{c}$ results in an increase in

Table 5. Values of amplitude, phase and the skin friction coefficients for different values of $\omega$ with $M=0.5, G_{c}=10$,

\begin{tabular}{ccccc}
$\boldsymbol{K}_{\boldsymbol{p}}=\mathbf{1 0}, \boldsymbol{\varepsilon}=\mathbf{0 . 0 0 2}, \boldsymbol{\omega}=\mathbf{5}$ and $\boldsymbol{\omega} \boldsymbol{t}=\frac{\boldsymbol{\pi}}{\mathbf{2}}$ & \\
\hline $\boldsymbol{\omega}$ & $\begin{array}{c}\text { Amplitude } \\
(|N|)\end{array}$ & $\begin{array}{c}\text { Phase } \\
(\tan (\alpha))\end{array}$ & $\tau$ & $\tau$ \\
\hline 5 & 15.6917 & -0.5289 & 18.9438 & 18.9585 \\
10 & 12.0945 & -0.7427 & 18.9438 & 18.9583 \\
15 & 10.2692 & -0.9189 & 18.9438 & 18.9577 \\
20 & 9.1398 & -1.0936 & 18.9438 & 18.9573 \\
\hline
\end{tabular}

Table 6. Values of amplitude, phase and the rate of mass transfer for different values of $S_{c}$ with $\varepsilon=0.002$ and $\omega t=\frac{\pi}{2}$.

\begin{tabular}{cccc}
\hline$S_{c}$ & Amplitude $(|Q|)$ & Phase $(\tan (\gamma))$ & $S_{h}$ \\
\hline 0.11 & 0.3345 & 0.2060 & 0.1097 \\
0.22 & 0.5333 & 0.2366 & 0.2194 \\
0.33 & 0.7218 & 0.2454 & 0.3289 \\
0.44 & 0.9115 & 0.2457 & 0.4384 \\
0.55 & 1.1064 & 0.2418 & 0.5476 \\
0.66 & 1.3089 & 0.2359 & 0.6567 \\
0.77 & 1.5197 & 0.2292 & 0.7654 \\
0.88 & 1.7396 & 0.2222 & 0.8741 \\
0.99 & 1.9687 & 0.2150 & 0.9824 \\
\hline
\end{tabular}

Table 7. Values of amplitude, phase and the rate of mass transfer for different values of $\omega$ with $\varepsilon=0.002$ and $S_{c}=\mathbf{0 . 2 2}$.

\begin{tabular}{cccc}
\hline$\omega$ & Amplitude $(|Q|)$ & Phase $(\tan (\gamma))$ & $S_{h}$ \\
\hline 5 & 0.5333 & 0.2366 & 0.2194 \\
10 & 0.6930 & 0.3118 & 0.2191 \\
15 & 0.8194 & 0.3454 & 0.2187 \\
20 & 0.9272 & 0.3651 & 0.2183 \\
\hline
\end{tabular}

$|Q|$ and $S_{h}$ but the phase $(\tan (\gamma))$ increases at $0.11 \leq$ $S_{c} \leq 0.44$ while it decreases at $S_{c} \geq 0.55$. An increase in $\omega$ results in an increase in $|Q|$ and $\tan (\gamma)$ and a decrease in $S_{h}$.

\section{Conclusions}

The above study shows up the following results of physical interest on the velocity and concentration distribution of the flow field.

- The magnetic field parameter $M$ slows down the velocity of the flow field at all points due to the magnetic pull of the Lorentz force acting on the flow field.

- The Schmidt number $S_{c}$ reveals that the presence of heavier diffusion species has a delaying effect on velocity of the flow field.

- The Grashof number for mass transfer $G_{c}$ is accelerated the velocity of the flow field at all points.

- The effect of porosity parameter $K_{p}$ is enhanced the velocity of the flow field at all points.

- The concentration distribution decreases at all points of the flow field with the increase of the Schmidt number $S_{c}$. It means that a greater delaying effect on the concentration distribution is due to the heavier diffusing species of the flow field.

- The value of the phase $(\tan (\alpha))$ increases due to decrease in $\omega$ while increases due to increase in $M$, $S_{c}, G_{c}$ and $K_{p}$.

- An increasing in $G_{c}$ and $K_{p}$ leads to an increasing in the value of amplitude $(|N|)$ while an increase of $M, S_{c}$ and $\omega$ leads to a decrease in the value of $|N|$.

- The skin friction coefficients $\tau_{\circ}$ and $\tau$ increase due to increase in $G_{c}, K_{p}$ while decrease due to increase in $M, S_{c}$ and $\omega$ with note that $\tau_{\circ}$ is not function on $\omega$.

- An increase in $S_{c}$ results in an increase in $|Q|$ and $S_{h}$ but the phase $(\tan (\gamma))$ increases at $0.11 \leq S_{c} \leq 0.44$ while it decreases at $S_{c} \geq 0.55$.

- An increase in $\omega$ results in an increase in $|Q|$ and $\tan (\gamma)$ and a decrease in $S_{h}$.

\section{REFERENCES}

[1] G. A. Georgantopoulos, J. Koullias, C. L. Goudas and C. Courogenis, "Free Convection and Mass Transfer Effects on the Hydro-Magnetic Oscillatory Flow past an Infinite Vertical Porous Plate," Astrophysics and Space Science, Vol. 74, No. 2, 1981, pp. 357-389. doi:10.1007/BF00656444

[2] T. Hayat, S. Ashgar and A. M. Siddiqui, "Periodic Unsteady Flows of a Non-Newtonian Fluid," Acta Mechanica, Vol. 131, No. 3-4, 1998, pp. 169-175.

[3] S. Ashgar, M. R. Mohyuddin, T. Hayat and A. M. Siddiqui, "The Flow of Non-Newtonian Fluid Induced Due to the Oscillations of a Porous Plate," Mathematical Problems in Engineering, Vol. 2, 2003, pp. 133-143. 
[4] P. Singh and C. B. Gupta, "MHD Free Convective Flow of a Viscous Fluid through a Porous Medium Bounded by an Oscillating Porous Plate in Slip Flow Regime with Mass Transfer," Indian Journal of Theoretical Physics, Vol. 53, No. 2, 2005, pp. 111-120.

[5] S. S. Das, S. K. Sahoo and G. C. Dash, "Numerical Solution of Mass Transfer Effects on Unsteady Flow past an Accelerated Vertical Porous Plate with Suction," Bulletin of the Malaysian Mathematical Sciences Society, Vol. 29, No. 1, 2006, pp. 33-42.

[6] A. Raptis, "Unsteady Free Convection Flow through Porous Medium," International Journal of Engineering Science, Vol. 21, No. 4, 1983, pp. 345-349. doi:10.1016/0020-7225(83)90118-0
[7] M. A. Hossain and R. A. Begum, "Effect of Mass Transfer and Free Convection on the Flow past a Vertical Plate," Journal of Heat Transfer, Vol. 106, 1984, pp. 664-668. doi:10.1115/1.3246735

[8] S. S. Das, M. Maity and J. K. Das, "Unsteady Hydromagnetic Convective Flow past an Infinite Vertical Porous Flat Plate in a Porous Medium," International Journal of Energy and Environment, Vol. 1, No. 3, 2012, pp. 109-118.

[9] A. K. Singh, A. K. Singh and N. P. Singh, "Heat and Mass Transfer in MHD Flow of a Viscous Fluid past a Vertical Plate under Oscillatory Suction Velocity," Indian Journal of Pure and Applied Mathematics, Vol. 34, No. 3, 2003, pp. 429-442.

\section{Nomenclatures}

\section{$C^{\prime} \quad$ Species concentration;}

C Non-dimension species concentration;

$D \quad$ Molecular diffusivity;

$G_{c} \quad$ Grashof number for mass transfer;

$G \quad$ Acceleration due to gravity;

$K^{\prime} \quad$ Permeability of the medium;

$K_{p} \quad$ Permeability/porosity parameter;

$M \quad$ Magnetic parameter;

$S_{c} \quad$ Schmidt number;

$t^{\prime} \quad$ Time;

$t \quad$ Non-dimension time;

$u^{\prime} \quad$ Velocity component along x-axis;

$u \quad$ Non-dimension velocity component along x-axis;

$v\left(t^{\prime}\right) \quad$ Suction velocity;

v. Positive constant suction velocity; $y^{\prime} \quad$ Distance along $y$-axis;

$y \quad$ Non-dimension distance along $y$-axis;

$\beta \quad$ Volumetric coefficient of expansion with species concentration;

$\rho$

$v$

$\omega^{\prime}$

$\omega$

$\tau$

$\sigma$

$\infty$

,

$S_{h} \quad$ Sherwood number. 


\section{Appendix}

$$
\begin{aligned}
& m_{1}=\frac{1}{2}\left[S_{c}+\sqrt{S_{c}^{2}+\mathrm{i} \omega S_{c}}\right]=A_{1}+\mathrm{i} B_{1}, \\
& m_{2}=\frac{1}{2}\left[1+\sqrt{1+4 a_{1}}\right], \\
& m_{3}=\frac{1}{2}\left[-1+\sqrt{1+4 a_{1}}\right], \\
& m_{4}=\frac{1}{2}\left[1+\sqrt{1+4 a_{2}}\right]=A_{2}+\mathrm{i} B_{2}, \\
& m_{5}=\frac{1}{2}\left[-1+\sqrt{1+4 a_{2}}\right]=A_{3}+\mathrm{i} B_{3}, \\
& \left(m_{4}-m_{1}\right)\left(m_{5}+m_{1}\right)=A_{4}+\mathrm{i} B_{4}, \\
& \left(m_{4}-S_{c}\right)\left(m_{5}+S_{c}\right)=A_{5}+\mathrm{i} B_{5}, \\
& \left(m_{4}-m_{2}\right)\left(m_{5}+m_{2}\right)=A_{6}+\mathrm{i} B_{6}, \\
& \left(m_{2}-S_{c}\right)\left(m_{3}+S_{c}\right)=a_{3}, \\
& A_{3}=A_{2}-1, \\
& A_{2}=\frac{1}{2}+\frac{1}{2 \sqrt{2}}\left[\sqrt{\sqrt{\left(1+4 a_{1}\right)^{2}+\omega^{2}}+1+4 a_{1}}\right], \\
& \frac{S_{c}}{2}\left[\sqrt{\sqrt{S_{c}^{2}+\omega^{2}}+S_{c}}\right],
\end{aligned}
$$$$
N_{3}=b_{1} \mathrm{e}^{-A_{1} y}\left\{\left(A_{4}-\frac{4 S_{c}}{\omega} B_{4}\right) \cos B_{1} y-\left(\frac{4 S_{c}}{\omega} A_{4}+B_{4}\right) \sin B_{1} y\right\}-b_{1} \mathrm{e}^{-A_{2} y}\left\{\left(A_{4}-\frac{4 S_{c}}{\omega} B_{4}\right) \cos B_{2} y-\left(\frac{4 S_{c}}{\omega} A_{4}+B_{4}\right) \sin B_{2} y\right\},
$$$$
N_{4}=b_{1} \mathrm{e}^{-A_{1} y}\left\{\left(\frac{4 S_{c}}{\omega} A_{4}+B_{4}\right) \cos B_{1} y+\left(A_{4}-\frac{4 S_{c}}{\omega} B_{4}\right) \sin B_{1} y\right\}-b_{1} \mathrm{e}^{-A_{2} y}\left\{\left(B_{4}+\frac{4 S_{c}}{\omega} A_{4}\right) \cos B_{2} y+\left(A_{4}-\frac{4 S_{c}}{\omega} B_{4}\right) \sin B_{2} y\right\} \text {, }
$$$$
N_{5}=b_{2} A_{5} \mathrm{e}^{-S_{c} y}-b_{2} \mathrm{e}^{-A_{2} y}\left\{A_{5} \cos B_{2} y-B_{5} \sin B_{2} y\right\},
$$$$
N_{6}=b_{2} B_{5} \mathrm{e}^{-S_{c} y}-b_{2} \mathrm{e}^{-A_{2} y}\left\{B_{5} \cos B_{2} y+A_{5} \sin B_{2} y\right\} \text {, }
$$$$
N_{7}=b_{3} B_{5} \mathrm{e}^{-S_{c} y}-b_{3} \mathrm{e}^{-A_{2} y}\left\{B_{5} \cos B_{2} y+A_{5} \sin B_{2} y\right\} \text {, }
$$$$
N_{8}=b_{3} \mathrm{e}^{-A_{2} y}\left\{A_{5} \cos B_{2} y-B_{5} \sin B_{2} y\right\}-b_{3} A_{5} \mathrm{e}^{-S_{c} y},
$$$$
N_{9}=b_{4} \mathrm{e}^{-A_{2} y}\left\{A_{6} \cos B_{2} y-B_{6} \sin B_{2} y\right\}-b_{4} A_{6} \mathrm{e}^{-m_{2} y} \text {, }
$$$$
N_{10}=b_{4} \mathrm{e}^{-A_{2} y}\left\{B_{6} \cos B_{2} y+A_{6} \sin B_{2} y\right\}-b_{4} B_{6} \mathrm{e}^{-m_{2} y},
$$$$
N_{11}=b_{5} A_{6} \mathrm{e}^{-m_{2} y}-b_{5} \mathrm{e}^{-A_{2} y}\left\{A_{6} \cos B_{2} y-B_{6} \sin B_{2} y\right\} \text {, }
$$$$
N_{12}=b_{5} B_{6} \mathrm{e}^{-m_{2} y}-b_{5} \mathrm{e}^{-A_{2} y}\left\{B_{6} \cos B_{2} y+A_{6} \sin B_{2} y\right\} \text {, }
$$$$
b_{1}=\frac{G_{c}}{A_{4}^{2}+B_{4}^{2}}
$$$$
b_{2}=\frac{G_{c}\left(\frac{1}{k_{p}}-S_{c}\right)}{a_{3}\left(A_{5}^{2}+B_{5}^{2}\right)},
$$$$
b_{3}=\frac{4 G_{c} S_{c}}{\omega\left(A_{5}^{2}+B_{5}^{2}\right)},
$$$$
b_{4}=\frac{G_{c}\left(\frac{1}{k_{p}}-m_{2}\right)}{a_{3}\left(A_{6}^{2}+B_{6}^{2}\right)},
$$$$
b_{5}=\frac{\left(\frac{1}{k_{p}}-m_{2}\right)}{A_{6}^{2}+B_{6}^{2}},
$$

$A_{5}=\left(A_{2}-S_{c}\right)\left(A_{3}+S_{c}\right)-B_{2}^{2}$,

$A_{6}=\left(A_{2}-m_{2}\right)\left(A_{3}+m_{2}\right)-B_{2}^{2}$,

$$
B_{1}=\frac{S_{c}}{2 \sqrt{2}}\left[\left[\sqrt{\sqrt{S_{c}^{2}+\omega^{2}}-S_{c}}\right]\right] \text {, }
$$$$
B_{2}=\frac{1}{2 \sqrt{2}}\left[\sqrt{\sqrt{\left(1+4 a_{1}\right)^{2}+\omega^{2}}-1-4 a_{1}}\right] \text {, }
$$$$
B_{3}=B_{2} \text {, }
$$$$
B_{4}=\left(A_{2}-A_{1}\right)\left(B_{2}+B_{1}\right)+\left(A_{3}+A_{1}\right)\left(B_{2}-B_{1}\right) \text {, }
$$$$
B_{5}=B_{2}\left(A_{2}+A_{3}\right) \text {, }
$$

$B_{6}=B_{5}$

$M_{r}=N_{1}+N_{3}+N_{5}+N_{7}+N_{9}+N_{11}$,

$M_{i}=-N_{2}-N_{4}-N_{6}-N_{8}-N_{10}-N_{12}$,

$L_{r}=\mathrm{e}^{-A_{1} y}\left(\cos B_{1} y-\frac{4 S_{c}}{\omega} \sin B_{1} y\right)$,

$L_{i}=\frac{4 S_{c}}{\omega} \mathrm{e}^{-S_{c} y}-\mathrm{e}^{-A_{1} y}\left(\frac{4 S_{c}}{\omega} \cos B_{1} y+\sin B_{1} y\right)$,

$N_{1}=\mathrm{e}^{-A_{2} y} \cos B_{2} y$,

$N_{2}=\mathrm{e}^{-A_{2} y} \sin B_{2} y$, 


$$
\begin{array}{rlr}
N_{r}= & b_{1}\left(A_{4}-\frac{4 S_{c}}{\omega} B_{4}\right)\left(A_{2}-A_{1}\right) & Q_{r}=A_{1}+\frac{4 S_{c}}{\omega} B_{1} \\
& +b_{1}\left(\frac{4 S_{c}}{\omega} A_{4}+B_{4}\right)\left(B_{2}-B_{1}\right)+B_{2}\left(b_{2} B_{5}-b_{3} A_{5}\right) & Q_{i}=B_{1}+\frac{4 S_{c}}{\omega}\left(S_{c}-A_{1}\right) . \\
& +B_{2} B_{5}\left(b_{5}-b_{4}\right)+\left(A_{2}-S_{c}\right)\left(b_{2} A_{5}+b_{3} B_{5}\right) & \\
& +A_{6}\left(A_{2}-m_{2}\right)\left(b_{5}-b_{4}\right)-A_{2}, \\
N_{i}= & b_{1}\left(A_{4}-\frac{4 S_{c}}{\omega} B_{4}\right)\left(B_{2}-B_{1}\right) \\
& -b_{1}\left(\frac{4 S_{c}}{\omega} A_{4}+B_{4}\right)\left(A_{2}-A_{1}\right)+B_{2}\left(b_{2} A_{5}+b_{3} B_{5}\right) \\
& +B_{2} A_{6}\left(b_{5}-b_{4}\right)+\left(A_{2}-S_{c}\right)\left(b_{3} A_{5}-b_{2} B_{5}\right) \\
& +B_{5}\left(A_{2}-m_{2}\right)\left(b_{4}-b_{2}\right)-B_{2},
\end{array}
$$

